\title{
Does research into contraceptive method discontinuation address women's own reasons? A critical review
}

\author{
Kumiyo Inoue, ${ }^{1}$ Alexandra Barratt, ${ }^{2}$ Juliet Richters ${ }^{3}$
}

\begin{abstract}
- Additional material is published online only. To view please visit the journal online (http://dx.doi.org/10.1136/fprhc2014-100976)

${ }^{1} \mathrm{PhD}$ Candidate, School of Public Health and Community Medicine, University of New South Wales, Sydney, Australia and Adjunct Senior Lecturer, School of Health Sciences, University of Tasmania, Hobart, Australia

${ }^{2}$ Professor, School of Public Health, University of Sydney, Sydney, Australia

${ }^{3}$ Professor, School of Public Health and Community Medicine, University of New South Wales, Sydney, Australia
\end{abstract}

\section{Correspondence to}

Dr Kumiyo Inoue, School of

Public Health and Community Medicine, University of New South Wales, High Street, Kensington, Sydney, New South Wales 2052, Australia; k.inoue@student.unsw.edu.au

Received 4 May 2014 Revised 30 August 2014 Accepted 3 December 2014 Published Online First 20 January 2015

\section{CrossMark}

To cite: Inoue K, Barratt A,
Richters J. J Fam Plann
Reprod Health Care
2015;41:292-299.

\begin{abstract}
Objective To examine the clinical and epidemiological literature addressing contraceptive method change or discontinuation and to assess whether the documented reasons reflected women's experiences.

Methods Major databases including Medline and PsycINFO were searched using keywords related to contraception and discontinuation, adherence and satisfaction, for articles published between January 2003 and February 2013.

Studies in developed countries that focused on women of reproductive age and reasons for method change or discontinuation were included. Reasons reported were categorised and examined.
\end{abstract}

Results A total of 123 papers were reviewed in detail. Medical terminology was generally used to describe reasons for method discontinuation.

The top two reported reasons were bleeding and pregnancy, but there was a lack of consensus about the categorisation of reasons. Broad categories that were not self-explanatory were included in more than half of the papers, often without further explanation. Only 12 studies expanded on categories containing 'other', 'non-medical' or 'personal' reasons. Eight papers included categories that attributed discontinuation to the participant, such as 'dissatisfied with method'.

Conclusions Studies of reasons for discontinuation of contraceptives do not well describe women's specific reasons. Studies rely heavily on medical terms and often fail to document women's subjective experiences. Future studies should create an opportunity for women to articulate their non-medical reasons in their own words, including those related to their sexual lives. Furthermore, researchers should distinguish, if possible, between reasons for discontinuation of a method and reasons for ceasing participation in a research study.
Key message points

- Women's points of view are interpreted within a biomedical framework in clinical studies.

- It is necessary to create an opportunity for women to articulate their reasons for discontinuation in their own words, including non-medical and sexual reasons.

- The category 'other' is a convenient category for researchers but can mask women's real reasons for discontinuation of contraceptives.

\section{INTRODUCTION}

Despite worldwide efforts to increase use of modern contraceptives, and to improve acceptability and therefore adherence, there is little consensus about women's reasons for changing methods or discontinuing use altogether. It has been argued that side effects are the most common reason why women discontinue contraceptive methods. ${ }^{1}{ }^{2}$ However, other researchers have found that some women who complained about oral contraceptives (OCs) and who subsequently discontinued using them had experienced no side effects or only mild ones. ${ }^{3}$ This suggests that women may be dissatisfied with contraceptives for reasons other than side effects. ${ }^{4}$

Clinical and epidemiological research addressing reasons for discontinuation of contraceptive methods could be expected to report this information from the women's point of view. However, clinical studies of contraceptive methods, framed by medical concerns, tend to measure variables such as effectiveness, safety, and 
adverse effects of contraceptives. Therefore we hypothesised that this framing may also extend to assessments of reasons for discontinuation.

In this study our aim was to examine the clinical and epidemiological literature addressing discontinuation of contraceptives to assess whether the documented reasons reflected women's experiences. We included studies in which women discontinued using any contraception as well as studies of women who changed from one contraceptive method to another.

\section{METHODS}

\section{Search strategy and screening}

Major databases (Medline, Scopus, EMBASE, PsycINFO, Informit and CINAHL) were searched using keywords related to contraception and discontinuation, adherence and satisfaction for papers published between January 2003 and February 2013 (see online Supplementary Material Appendix 1 for details of the search strategy used in each database). Furthermore, papers recommended by the database function as being 'related' were also considered and retained for further examination. Titles and abstracts of the searched papers were screened and selected papers were exported to an Endnote library, with duplicates being identified and excluded (see online Supplementary Material Appendix 2 for details of the inclusion and exclusion criteria).

\section{Data entry and data analysis}

Reasons reported in each paper for discontinuation of contraceptives were extracted and imported into the qualitative research software package NVivo $10^{\mathrm{TM}}$ (QSR International). Each reason for discontinuation was made into an NVivo node (index term) without changing the wording. The term 'node' is synonymous with 'code' as used in this article, namely "a word or short phrase that symbolically assigns a summative, salient, essence-capturing and/or evocative label for a piece of textual data". ${ }^{5}$ We used 'in vivo' coding to keep the analysis rooted in the language used in the literature under review.

Nodes with identical wording were merged and similar nodes (such as 'wish to become pregnant' and 'wish to conceive') were gathered under a 'parent' node (such as 'Pregnancy'). Parent nodes contained subnodes ('planned', 'unplanned' and 'intention unknown' in this example), differentiating where possible between discontinuation for a reason related to women's preference and discontinuation because of method failure. This approach allowed us to record and analyse the variety of expressions used to describe reasons for discontinuation.

\section{RESULTS}

A total of 4535 citations were retrieved using the aforementioned search strategies (see online Supplementary Material Appendix 1). Duplicate titles and irrelevant papers were excluded. Thirty-five articles obtained from the 'related document' function were added. The titles and abstracts of the remaining 627 papers were screened further, with detailed application of the inclusion and exclusion criteria, leaving 186 papers which all discussed discontinuation. Further examination of the complete texts excluded papers reporting duplicate results and studies that gave no reasons for discontinuation, resulting in 123 papers being suitable for analysis.

\section{Topics and study designs}

Most studies addressed hormonal contraceptives such as oral contraceptive pills, the vaginal ring, implants, injectables, and hormone-releasing intrauterine devices (IUDs) and patches. The studies examined various aspects of contraceptives and their usage such as: efficacy and failure rates, adherence, compliance, acceptability, satisfaction, safety, (dis)advantages, side effects, cycle control, bleeding characteristics and reasons for (dis)continuation. Some studies compared specific pairs of contraceptive methods such as the ring and the patch or the ring and the pill. Only a few papers compared a wide variety of contraceptive methods, including copper IUDs, spermicides, condoms, cervical barriers, withdrawal and fertility awareness methods. Other studies investigated different regimens or bleeding patterns. Study designs and methods were well documented. Sixty-one experimental studies, 60 observational studies and two qualitative studies were found. The 61 experimental studies included 32 randomised trials. Many of the papers included a specific study description such as 'multicentre clinical study' or 'multicentre, open label, single arm, clinical study'. The observational studies included prospective and retrospective cohort studies, cross-sectional studies and chart reviews. One of the two qualitative studies used a focus group approach and the other collected data through one-on-one interviews.

\section{Categories of discontinuation reasons}

Thirty-one parent nodes were categorised from a total of 789 nodes (see online Supplementary Material Appendix 3). Of these, the node termed Bleeding $(n=88)$ was the most reported reason for discontinuation followed by Pregnancy $(n=62)$, Other $(n=51)$, Pain $(n=47)$, Compliance issues $(n=43)$, Adverse events $(n=42), \quad$ Side effects $(n=35)$ and Participant-related issues (i.e. 'became menopausal', 'individual patient's decision', 'subject's request', 'not willing to cooperate', and so on) $(n=38)$. The numbers in parentheses refer to the number of papers that included each stated reason for discontinuation.

The parent node Participant-related issues contains categories stating this fact but authors did not often explain the reasons. For example, the category 'individual patient's decision' (to discontinue the method) 
states what happened but does not shed light on the participant's reason for discontinuation. When adverse events were reported together with detailed information in brackets $(n=7)$, such as 'adverse events' (bleeding, increased weight, etc.), all the reasons in the brackets were recoded into relevant nodes. When a category called 'Other' detailed the information that constituted it, all the reasons in the brackets were recoded into relevant nodes.

Some studies reported percentages of those in the discontinuation subsample while others calculated them based on the entire sample included in the data analysis. For example, the percentage of women whose reasons for discontinuation fell into the category of 'other' ranged from $0.4 \%$ to $78 \%$ in the studies reviewed. Only one paper clearly addressed what the percentages referred to, stating that $4 \%$ of all users discontinued their participation in the study for 'other reasons'. ${ }^{6}$

\section{The top two reasons: bleeding and pregnancy}

'Bleeding' included changes in bleeding patterns, the amount of bleeding (e.g. excessive or heavy bleeding), nature of bleeding (e.g. spotting and irregular bleeding) and frequency of bleeding. Some papers used medical terminology (e.g. amenorrhoea, dysmenorrhoea and menorrhagia). Bleeding was referred to as both 'periods' and 'menstruation'. Some studies included specific information such as 'vaginal bleeding' and 'withdrawal bleeding'. Only a few studies tried to grasp women's subjective experiences of bleeding, using terms such as 'unacceptable bleeding', 'nuisance bleeding' and 'intolerance to the symptom of amenorrhoea'. It was often unclear whether these terms were used by the women themselves or determined by the researchers. Not all of the studies included a clear definition of bleeding but some papers adopted the bleeding profiles defined by the World Health Organization (WHO) to differentiate amongst bleeding, spotting, bleeding day, spotting day, bleeding-free day, bleeding/spotting episode, bleeding episode and amenorrhoea.

There were discrepancies between women's and investigators' perceptions of bleeding. Archer et al. ${ }^{8}$ reported that subjects who requested withdrawal from the study comprised $15.7 \% \quad(n=335)$ of those sampled and included those who cited reasons related to uterine bleeding events ( $9.8 \%$ of all subjects). However, they did not have clinically important uterine bleeding in the opinion of the investigator and were not reported by the investigator as 'uterine bleeding as an adverse event causing withdrawal' from the study. ${ }^{8}$ Anderson and Hait $^{9}$ reported that unacceptable bleeding was sometimes cited as an adverse event but at other times cited as simply the reason given by an individual for her decision to withdraw from the study.
Some women discontinued using contraceptives because they wished to conceive whereas others encountered an unintended pregnancy. Terms such as 'desired pregnancy' or 'planned conception' were used in some papers but others simply gave 'became pregnant' or 'pregnancy' as reasons and so it was unclear whether or not this indicated a planned pregnancy.

\section{'Other' as reasons for discontinuation of contraceptives}

The category 'other' was variously defined by researchers. It sometimes consisted of unknown reasons but not always. Small studies and those reporting a small number of discontinuations provided detailed information about what constituted this category. Studies focused on specific conditions such as weight gain and bleeding often specified how many women discontinued and for what reasons. In contrast, large studies including different contraceptive methods ${ }^{10}$ and/or data collected from multiple sites ${ }^{11}$ tended to categorise reasons for discontinuation broadly into 'other' without explanation. Some researchers ${ }^{12}$ even put pregnancy into the category 'other' whereas other researchers ${ }^{13}$ considered that pregnancy was due to user failure and categorised it as non-compliance.

One-third (41/123) of the papers included the term 'other' alone or in combination with other words ['other reasons', 'other personal', 'another reason', 'unknown/other', and '(an)other method'] as a reason for method discontinuation. A further 27 papers included the terms 'non-medical', 'unknown', 'personal', 'personal reasons', 'unspecified reasons', 'unwilling to continue', 'subject request', 'any termination', 'user-related', 'patient's own decision', 'patient's choice', 'switch to another contraceptive' or 'no reasons elicited'. There were only 12 papers in which the category 'other' was expanded by detailed information outlining what constituted the other reasons.

Eight papers did not include any category such as 'other' or 'unknown' but included categories that attributed discontinuation to the participant, such as 'premature discontinuation of study medication', 'early termination', 'did not complete survey', 'early withdrawal', 'dissatisfied with contraceptive method', 'used other contraceptive method' and 'did not really want it'. Some papers used both 'other' and also 'nonmedical reasons', and thus the above-listed reasons overlapped in some studies. As a result, nearly two-thirds of the 123 papers used at least one of the above-mentioned ambiguously addressed categories as reasons for discontinuation.

In one paper ${ }^{14}$ the authors reported two specific reasons for discontinuation of either condoms or OCs ('not having sex' and 'trying to get pregnant'), and remaining reasons were all gathered into the category 'other'. The women were given the opportunity to 
elaborate in more detail on their reasons. In the case of condoms, they referred to issues such as a desire to switch to an easier or more reliable method, a monogamous relationship, the perception that condoms were uncomfortable and interfered with their sex life, vaginal irritation and latex allergies. In the case of OCs they mentioned issues such as yeast infections, nausea, episodes of depression, headaches, the development of hypertension and breakthrough bleeding.

Other authors clearly addressed how they categorised the category 'other'. Harvey et al. ${ }^{15}$ noted that when women reported single reasons for discontinuation, such as low libido, pelvic pain, headaches, mastalgia, arm pain, dislike of hormones or light menstrual flow, these were combined under the category 'other'. This is understandable because the researchers were specifically interested in rates of and reasons for removal of the implant. Nevertheless, it is possible that many of the reasons for discontinuation were overlooked. The participants were using one of 13 contraceptive methods, so their reasons for discontinuation would have been varied and method-specific.

Some studies regarded switching to another method as discontinuation, but other studies did not, or were unclear. One example was a retrospective study on IUDs in which the category 'other' included 'subject using another birth control method' but this was not fully explored. ${ }^{16}$ 'Other' reasons presented by another study included 'fear of hormone/cancer', ${ }^{17}$ which is an example of women's subjective claims being gathered into the category 'other'.

One study on the IUD provided detailed information about each category, including what constituted the category 'other'. ${ }^{18}$ Only two subjects belonged to the category 'other' in this study and only one of them gave her reason for the removal of the IUD. Another one of the categories was 'pelvic inflammatory disease', and seven women were reported to have been treated for this condition, which required the removal of the IUD. Their own opinion of the IUD was not recorded in the paper.

\section{Pain}

A variety of expressions describing pain were found, such as 'headache', 'migraine', 'breast pain', 'mastalgia', 'pelvic pain', 'abdominal pain', 'arm pain', 'cramping' and 'general pain', but some of the papers merely stated 'pain' without explaining the location or type of pain.

\section{Compliance}

The category '(non)compliance' was generally more explicit, with reasons given such as difficulty remembering, loss of health insurance, lack of transport, logistical reasons, lack of time, and difficulty keeping track of the ring.
No longer in need of contraception

Ten studies used the terms 'end of relationship', 'no longer in a relationship', 'no longer sexually active' or 'no longer in need of contraceptives'. These expressions have slightly different nuances but are not explored in any depth in the studies. As noted above, pregnancy during method use and discontinuation to become pregnant were sometimes recorded as reasons for no longer needing contraception.

\section{Adverse effects and side effects}

The terms 'adverse events' and 'side effects' were used to describe medically undesired signs or symptoms associated with a method, but were used inconsistently. Only Winkler et al. ${ }^{19}$ listed all of the adverse events recognised by the $\mathrm{WHO}$ in a table and reported the number of women who experienced each. The most frequently addressed adverse event was bleeding but, as noted above, the degree and nature of bleeding was not always clear.

\section{Loss to follow-up and withdrawal of consent}

Eighty-eight of the $123(72 \%)$ studies reviewed were either clinical trials or cohort studies and approximately one-third of the studies (36) provided the number of participants who were lost to follow-up. Generally, the 'lost to follow-up' group was reported separately from the reasons for discontinuation $(n=19)$, but many studies $(n=17)$ that included the category 'lost to follow-up' referred to this as a reason for discontinuation. For example, one paper reported that 'the most common reasons for premature discontinuation were adverse events, lost [sic] to follow-up, and individual patient decision. ${ }^{9}$ Clearly, however, it is not possible to know whether these participants continued using the given contraceptive method or not, since the investigators were unable to follow them up. The percentage of those lost to follow-up was occasionally as high as $50 \%$ of the subjects reported as having discontinued a method. ${ }^{12}$ Some studies reported 'withdrawal of consent' as a separate issue from reasons for discontinuation; however, two studies included 'withdrawal of consent' as a reason for discontinuation of the study. For example, a Phase 3, open-label, non-comparator, multinational, multicentre 1-year study grouped reasons for discontinuation into four categories: 'adverse events', 'withdrawal of consent', 'loss to follow-up' and 'protocol violation'. ${ }^{20}$

Apart from adverse events, it is unclear why some women withdrew their consent or failed to attend follow-up sessions. Another ambiguous categorisation was found in a study that included not only 'withdrawal of consent' as one of the reasons for discontinuation of the study but also a category called 'premature discontinuation of the study medication'. ${ }^{21}$ In this case, 'withdrawal of consent' could possibly be interpreted as withdrawal of consent with no reasons 
elicited and 'premature discontinuation of the study medication' might be interpreted as withdrawal from the study due to dissatisfaction with the study medication. All of these categorisations such as 'withdrawal of consent', 'loss to follow-up', 'protocol violation' and 'premature discontinuation of the study medication' are expressed from the researchers' point of view, merely reporting the fact that some women discontinued participating in the study, without exploring or citing the reasons given by the women themselves. Some women may have chosen to withdraw their consent or no longer participate in the study (and were therefore lost to follow-up) without explanation. Others may have given an explanation that was not recorded.

\section{Diverse categorisation of reasons for discontinuation}

Overall, the categorisations reflected a lack of definition and agreement as well as great diversity about what constituted discontinuation. Many reported from the most common to the least common reasons. ${ }^{15}$ A few studies reported only the most commonly addressed reasons. ${ }^{22-25}$ For example, Brucker et al.'s ${ }^{26}$ study reported only the main reason cited by women for their discontinuation. As the most commonly reported reason was side effects $(35 \%)$, reasons given by the other $65 \%$ of the women were not reported in the paper. This tendency was more apparent when the scope of the study was very narrow, for example a study ${ }^{27} 28$ that focused on adverse mood effects, and therefore reported only two categories explaining discontinuation, 'adverse mood effects' and 'other than adverse mood effects'. It was evident that some researchers were unable to obtain reasons: one study $^{29}$ failed to obtain reasons for discontinuation from nearly $40 \%$ of women who discontinued IUDs.

\section{Do studies reflect the voices of women or researchers?}

Some studies strove to obtain detailed information about women's reasons for discontinuation by asking open-ended questions ${ }^{3}{ }^{30}$ and/or allowing for multiple reasons to be noted by participants. It was generally unclear whether women had been given the option to provide multiple responses. Exceptions were Rai et al. $^{31}$ who noted how many implant removals had multiple indications, and Moreau et al. ${ }^{4}$ who allowed up to 14 reasons for women to address. Considering the broad scope of the latter study, which focused retrospectively on contraceptive discontinuation over women's lives due to dissatisfaction, allowing women to address up to 14 reasons is reasonable. It is unrealistic to expect that women will have used a single contraceptive throughout their life unless they are still at the early stage of contraceptive use. In fact, Moreau et al. reported that "the proportion of women who had ever discontinued a method due to dissatisfaction increased with the number of different methods ever used. While a quarter of women who used fewer than three different methods had discontinued at least one of them due to dissatisfaction, $80 \%$ of those who used five or more different methods had discontinued for this reason". 4 Even in this study, the category 'other reason' accounted for between $8 \%$ and $16 \%$ of reasons for discontinuation $(8 \%$ for depot medroxyprogesterone injections, $10 \%$ for implants, $11 \%$ for the pill and $16 \%$ for condoms). However, more importantly, the two reasons 'experienced side effects' and 'worried about side effects' together constituted one-fifth of the reasons for discontinuation of condoms and over three-quarters for hormonal contraceptives such as the pill (80\%), implants (75\%) and depot injections (77\%).

Except for the studies offering an open-ended questionnaire $^{3} 30$ that allowed participants to address issues in their own words, the majority of the studies did not report how the reasons for discontinuation were obtained. If women were asked to choose their reasons for discontinuation of contraceptives from a list of fixed (medical) reasons, they could have chosen the category 'other' either passively because they could not articulate a reason, or deliberately because their own reason was not listed.

\section{Dissociation between contraceptives and sex}

Some studies used the category 'other' to describe reasons such as 'no longer having a sexual relationship' and 'relationship ended', whereas other studies did not gather those reasons into the category of 'other'. Regardless of how these reasons are categorised, it is an inevitable argument that the use of contraceptives is directly linked to having sexual intercourse, with the possible exception of some contraceptive pills used as treatment for acne or for cycle control. Side effects such as bleeding, spotting, mood change, headaches, nausea and weight gain are not directly related to sexual intercourse, but they may directly and/or indirectly affect sexual life.

Five studies reported decreased (or low or loss of) libido as one of the reasons for discontinuation of contraceptive methods. Four of them created a separate category describing changes in libido (e.g. 'decreased libido' and 'asthenia'), ${ }^{32-36}$ but one gathered low libido into the category 'other' together with other reasons such as pelvic pain, headaches, mastalgia, arm pain, dislike of hormones and light menstrual flow. ${ }^{10}$ It is unclear whether 'libido' is a term actually used by women or whether researchers use this term to describe what the women reported. As it is predominantly a medical and not a colloquial term, it is unlikely that it has a shared meaning between health care professionals and women themselves.

Other possible sex-related reasons for method discontinuation such as physical discomfort (e.g. from a diaphragm or vaginal ring, or from an IUD during deep penetration) or partner discomfort (e.g. from an IUD string) were notably absent from the study 
reports. This could be due to women's unwillingness to talk explicitly about sex in the clinical context, as this is not something people do in their everyday life. Women may not be confident that they have the appropriate language to describe their experiences. ${ }^{37} 38$ Women may take advantage of the availability of medical terms instead of revealing personal information using everyday language. Alternatively they may offer no response if confronted with a word or term they do not understand. ${ }^{39}$ Settling for apparently objective categories such as 'adverse events', 'side effects' and even 'bleeding' allows researchers to avoid asking women to address sexual issues directly.

\section{DISCUSSION}

This is the first comprehensive review of the categorisation of reasons for contraceptive discontinuation as reported in published literature. Our focus was to explore how women's voices were treated. Quite often, in studies to date, women's own experiences have been replaced by scientific language or the interpretation of researchers. ${ }^{40}$ Related to this there can sometimes be a discrepancy between women's perceptions and investigators' perceptions. An example of this can be found in Archer et al.'s study ${ }^{8}$ in which some women reported what they perceived to be problematic bleeding events. These, however, were not always judged by the investigators to be of clinical importance. Although this approach is appropriate in a clinical study focusing on clinically defined adverse events, women's own voices cannot reasonably be translated from the results of clinical studies such as this one. In a qualitative study the most meaningful and relevant judgment about whether or not a particular contraceptive method is acceptable or not comes from the users themselves.

Researchers had unique ways of categorising not only reasons for discontinuation but also the category 'other', which could encompass a variety of reasons. It sometimes consisted of unknown reasons but this was not always the case. When studies used a small sample size and/or only a small number of discontinuations was reported, detailed information was provided about what constituted this category. When a study focused on specific conditions such as weight gain and bleeding, reasons for discontinuation were often specific, including how many women discontinued and for what reasons. In contrast, when the scope of the study was extensive, including different types of contraceptive methods ${ }^{16}$ and/or the data were collected from multiple sites, ${ }^{11}$ reasons for discontinuation tended to be categorised broadly into 'other' without explanation. Some researchers ${ }^{12}$ even put pregnancy into the category 'other' whereas other researchers ${ }^{13}$ considered that pregnancy was due to user failure and categorised it as non-compliance.

It is unclear from the information provided in most of the papers reviewed whether women were asked their reasons for discontinuation or were simply allocated to a category by the health care provider or researchers. Some researchers asked open-ended questions in their written surveys but it is unclear whether they were actually investigating women's experiences. Open-ended questions attached to some surveys may extract some idiosyncratic responses but are unlikely to explore the impact of contraceptives on women and their lives in depth.

Furthermore, these are clinical conditions such as adverse events and side effects that do not necessarily reflect the complexities of women's voices. These studies might be useful for ascertaining the proportion of women who discontinue use of methods, but they do not investigate women's subjective points of view. A recent UK study ${ }^{41}$ of the implant demonstrates the complexity of women's discourse about irregular bleeding. They assert that women's experiences of irregular bleeding cannot be understood simply in terms of irregularity or 'extensive bleeding'. Bleeding can also impact on women's sex lives and be accompanied by pain, exhaustion, embarrassment as a result of leaking due to unpredictable bleeding patterns and even 'sensitivity towards the smell of bleeding'. Clearly women's varied and unique experiences of irregular bleeding cannot be adequately described by the simple expression 'irregular bleeding'.

This study has some limitations. Non-English papers requiring translation were excluded due to financial constraints. Except for the two qualitative research papers identified in this review, there might be other studies of contraceptive use not addressing discontinuation directly, but still examining women's reasons, feelings or experiences in more depth than the ones reviewed here.

\section{CONCLUSIONS}

It is unilluminating to attempt to understand women's contraceptive experiences from clinical studies looking at (dis)continuation of contraceptives, as their voices and subjective experiences are not well captured and reported. The wording in contraceptive research is also important because the use of contraceptives always involves the sexual lives of the women who use them. We recommend that researchers studying women's acceptance and/or discontinuation of contraceptive methods examine women's own stated reasons for discontinuation in more detail. This information would be very useful for scientists developing better contraceptives. Capturing and reporting the precise reasons why women abandon specific contraceptive modalities is of utmost importance, since clinicians, governmental and local health care providers and regulatory authorities base their clinical and financial decisions on whether to introduce or retain a specific contraceptive product on many factors including its acceptability to consumers. In any study, the two categories 'lost to follow-up' and 'withdrawal of consent' 
(to participation in the study) should be differentiated from reasons for discontinuation of contraceptives. It is often unknown whether the women in these groups discontinued contraceptives or not, let alone what their reasons were.

Studies dedicated to understanding women's reasons for discontinuation are much needed and a qualitative approach that focuses on the voices of women is most appropriate for this type of investigation. New research in this area would lead to better categories for coding women's responses in epidemiological and clinical research. Embedding the voice of women in clinical research is challenging because the philosophical underpinnings of clinical research and qualitative research are so different. Researchers and clinicians should acknowledge the benefits of each type of study and clinical researchers must seek a person-centred approach if they are to begin to fill the gap between what women actually want from contraceptives and the current contraceptive options and services available.

\section{Competing interests None.}

Provenance and peer review Not commissioned; externally peer reviewed.

\section{REFERENCES}

1 Rosenberg MJ, Waugh MS. Oral contraceptive discontinuation: a prospective evaluation of frequency and reasons. Am J Obstet Gynecol 1998;179:577-582.

2 Sanders SA, Graham CA, Bass JL, et al. A prospective study of the effects of oral contraceptives on sexuality and well-being and their relationship to discontinuation. Contraception 2001;64:51-58.

3 Westhoff CL, Heartwell S, Edwards S, et al. Oral contraceptive discontinuation: do side effects matter? Am J Obstet Gynecol 2007;196:412.e1-412.e7.

4 Moreau C, Cleland K, Trussell J. Contraceptive discontinuation attributed to method dissatisfaction in the United States. Contraception 2007;76:267-272.

5 Saldana J. The Coding Manual for Qualitative Researcher. Thousand Oaks, CA: Sage Publications, 2008.

6 Merki-Feld GS, Hund M. Clinical experience with NuvaRing ${ }^{\circledR}$ in daily practice in Switzerland: cycle control and acceptability among women of all reproductive ages. Eur J Contracept Reprod Health Care 2007;12:240-247.

7 Stewart FH, Kaunitz AM, Laguardia KD, et al. Extended use of transdermal norelgestromin/ethinyl estradiol: a randomized trial. Obstet Gynecol 2005;105:1389-1396.

8 Archer DF, Jensen JT, Johnson JV, et al. Evaluation of a continuous regimen of levonorgestrel/ethinyl estradiol: phase 3 study results. Contraception 2006;74:439-445.

9 Anderson FD, Hait H. A multicenter, randomized study of an extended cycle oral contraceptive. Contraception 2003;68:89-96.

10 Lete I, Pérez-Campos E, Correa M, et al. Continuation rate of combined hormonal contraception: a prospective multicenter study. J Women's Health 2012;21:490-495.

11 Matsumoto Y, Yamabe S, Sugishima T, et al. Perception of oral contraceptives among women of reproductive age in Japan: a comparison with the USA and France. J Obstet Gynaecol Res 2011;37:887-892.
12 Edelman AB, Koontz SL, Nichols MD, et al. Continuous oral contraceptives: are bleeding patterns dependent on the hormones given? Obstet Gynecol 2006;107:657-665.

13 Bachmann G, Sulak PJ, Sampson-Landers C, et al. Efficacy and safety of a low-dose 24-day combined oral contraceptive containing 20 micrograms ethinylestradiol and $3 \mathrm{mg}$ drospirenone. Contraception 2004;70:191-198.

14 Brunner Huber LR, Hogue CJ, Stein AD, et al. Contraceptive use and discontinuation: findings from the contraceptive history, initiation, and choice study. Am J Obstet Gynecol 2006;194:1290-1295.

15 Harvey C, Seib C, Lucke J. Continuation rates and reasons for removal among Implanon ${ }^{\circledR}$ users accessing two family planning clinics in Queensland, Australia. Contraception 2009;80:527-532.

16 Lete I, Del Carme Cuesta M, Marín JM, et al. Acceptability of the levonorgestrel intrauterine system in the long-term treatment of heavy menstrual bleeding: how many women choose to use a second device? Eur J Obstet Gynecol Reprod Biol 2011;154:67-70.

17 Caruso S, Agnello C, Intelisano G, et al. Prospective study on sexual behavior of women using 30 microg ethinylestradiol and $3 \mathrm{mg}$ drospirenone oral contraceptive. Contraception 2005;72:19-23.

18 Drey EA, Reeves MF, Ogawa DD, et al. Insertion of intrauterine contraceptives immediately following first- and second-trimester abortions. Contraception 2009;79:397-402.

19 Winkler UH, Ferguson H, Mulders JAPA. Cycle control, quality of life and acne with two low-dose oral contraceptives containing $20 \mu \mathrm{g}$ ethinylestradiol. Contraception 2004;69:469-476.

20 Jain J, Jakimiuk AJ, Bode FR, et al. Contraceptive efficacy and safety of DMPA-SC. Contraception 2004;70:269-275.

21 Heger-Mahn D, Warlimont C, Faustmann T, et al. Combined ethinylestradiol/gestodene contraceptive patch: two-center, open-label study of ovulation inhibition, acceptability and safety over two cycles in female volunteers. Eur J Contracept Reprod Health Care 2004;9:173-181.

22 Deokar AM, Jackson W, Omar HA. Menstrual bleeding patterns in adolescents using etonogestrel (ENG) implant. Int J Adolesc Med Health 2011;23:75-77.

23 Rickert VI, Tiezzi L, Lipshutz J, et al. Depo now: preventing unintended pregnancies among adolescents and young adults. J Adolesc Health 2007;40:22-28.

24 Thurman AR, Hammond N, Brown HE, et al. Preventing repeat teen pregnancy: postpartum depot medroxyprogesterone acetate, oral contraceptive pills, or the patch? J Pediatr Adolesc Gynecol 2007;20:61-65.

25 Thomas AG, Klihr-Beall S, Siqueira L, et al. Concentration of depot medroxyprogesterone acetate and pain scores in adolescents: a randomized clinical trial. Contraception 2005;72:126-129.

26 Brucker C, Karck U, Merkle E. Cycle control, tolerability, efficacy and acceptability of the vaginal contraceptive ring, NuvaRing: results of clinical experience in Germany. Eur J Contracept Reprod Health Care 2008;13:31-38.

27 Borgström A, Odlind V, Ekselius L, et al. Adverse mood effects of combined oral contraceptives in relation to personality traits. Eur J Obstet Gynecol Reprod Biol 2008;141:127-130.

28 Borgström A, Kask K, Gulinello M, et al. Patients with adverse mood effects from combined oral contraceptives have lower levels of prepulse inhibition than healthy controls. Psychoneuroendocrinology 2008;33:487-496. 
29 Teal SB, Sheeder J. IUD use in adolescent mothers: retention, failure and reasons for discontinuation. Contraception 2012;85:270-274.

30 Ersek JL, Brunner Huber LR, Thompson ME, et al. Satisfaction and discontinuation of contraception by contraceptive method among university women. Matern Child Health J 2011;15:497-506.

31 Rai K, Gupta S, Cotter S. Experience with Implanon ${ }^{\circledR}$ in a north-east London family planning clinic. Eur J Contracept Reprod Health Care 2004;9:39-46.

32 Short M, Dallay D, Omokanye S, et al. Acceptability of the levonorgestrel releasing-intrauterine system and etonogestrel implant: one-year results of an observational study. Eur J Contracept Reprod Health Care 2012;17:79-88

33 Oddsson K, Leifels-Fischer B, Wiel-Masson D, et al. Superior cycle control with a contraceptive vaginal ring compared with an oral contraceptive containing 30 microg ethinylestradiol and 150 microg levonorgestrel: a randomized trial. Hum Reprod 2005;20:557-562.

34 Oddsson K, Leifels-Fischer B, de Melo NR, et al. Efficacy and safety of a contraceptive vaginal ring (NuvaRing) compared with a combined oral contraceptive: a 1-year randomized trial. Contraception 2005;71:176-182.
35 Gezginc K, Balci O, Karatayli R, et al. Contraceptive efficacy and side effects of Implanon ${ }^{\circledR}$. Eur J Contracept Reprod Health Care 2007;12:362-365.

36 Warner P, Guttinger A, Glasier AF, et al. Randomized placebocontrolled trial of CDB-2914 in new users of a levonorgestrelreleasing intrauterine system shows only short-lived amelioration of unscheduled bleeding. Hum Reprod 2010;25:345-353.

37 Fennell J. 'And isn't that the point?' Pleasure and contraceptive decisions. Contraception 2014;89:264-270.

38 Hayfield N, Clarke V. 'I'd be just as happy with a cup of tea': women's accounts of sex and affection in long-term heterosexual relationships. Women Stud Int Forum 2012;35:67-74.

39 Binson D, Catania JA. Respondents' understanding of the words used in sexual behavior questions. Public Opin $Q$ 1998;62:190-208.

40 Heise LL. Beyond acceptability: reorienting research on contraceptive choice. In: Ravindran TKS, Berer M, Cottingham J (eds), Beyond Acceptability: Users' Perspectives on Contraception. London, UK: Reproductive Health Matters for the World Health Organization, 1997:6-14.

41 Hoggart L, Newton VL, Dickson J. 'I think it depends on the body, with mine it didn't work': explaining young women's decisions to request subdermal contraceptive implant removal. Contraception 2013;88:636-640. 